\title{
MiR-27b Promotes Muscle Development by Inhibiting MDFI Expression
}

\author{
Lianjie Hou Jian $\mathrm{Xu}^{\mathrm{a}}$ Yiren Jiao ${ }^{\mathrm{a}}$ Huaqin $\mathrm{Li}^{\mathrm{a}}$ Zhicheng Pan ${ }^{\mathrm{a}}$ Junli Duan \\ Ting $\mathrm{Gu}^{\mathrm{a}}$ Chingyuan $\mathrm{Hu}^{\mathrm{b}}$ Chong Wanga
}

aNational Engineering Research Center for Breeding Swine Industry, Guangdong Provincial Key Lab of Agro-Animal Genomics and Molecular Breeding, College of Animal Science, South China Agricultural University, Guangzhou, PRC, 'D Department of Human Nutrition, Food and Animal Sciences, College of Tropical Agriculture and Human Resources, University of Hawaii at Manoa, Honolulu, USA

\section{Key Words}

MiR-27b • MDFI • Skeletal muscle • Porcine • Satellite cells

\begin{abstract}
Background/Aims: Skeletal muscle plays an essential role in the body movement. However, injuries to the skeletal muscle are common. Lifelong maintenance of skeletal muscle function largely depends on preserving the regenerative capacity of muscle. Muscle satellite cells proliferation, differentiation, and myoblast fusion play an important role in muscle regeneration after injury. Therefore, understanding of the mechanisms associated with muscle development during muscle regeneration is essential for devising the alternative treatments for muscle injury in the future. Methods: Edu staining, qRT-PCR and western blot were used to evaluate the miR-27b effects on pig muscle satellite cells (PSCs) proliferation and differentiation in vitro. Then, we used bioinformatics analysis and dual-luciferase reporter assay to predict and confirm the miR-27b target gene. Finally, we elucidate the target gene function on muscle development in vitro and in vivo through Edu staining, qRT-PCR, western blot, H\&E staining and morphological observation. Result: miR-27b inhibits PSCs proliferation and promotes PSCs differentiation. And the miR-27b target gene, MDFI, promotes PSCs proliferation and inhibits PSCs differentiation in vitro. Furthermore, interfering MDFI expression promotes mice muscle regeneration after injury. Conclusion: our results conclude that miR-27b promotes PSCs myogenesis by targeting MDFI. These results expand our understanding of muscle development mechanism in which miRNAs and genes work collaboratively in regulating skeletal muscle development. Furthermore, this finding has implications for obtaining the alternative treatments for patients with the muscle injury.

\section{Introduction}

Skeletal muscle comprises about $45 \%$ of the human body mass, and the musculoskeletal system injuries are common. Mechanical muscle damage often accompanied by muscle swelling, atrophy, contracture, and even functional disorders [1, 2]. Understanding of the 
Hou et al.: MiR-27b Promotes Muscle Development through MDFI

mechanisms involved in muscle development is critical in identifying treatments for these patients in the future.

Muscle satellite cells, also known as muscle stem cells, reside as quiescent cells beneath the basal lamina that surrounds muscle fibers, and function as myogenic precursors for muscle growth and repair [3,4]. Satellite cell culture is a useful tool, and it has been used extensively to study muscle development in vitro [5]. Many myogenic markers during muscle development were identified in the past several decades. For example, $p 21$ and cyclins are well-established proliferation markers, and $\mathrm{MyoD}, \mathrm{MyHC}$, and myosin are the differentiation markers [6-8].

$M y o D$ is one of the earliest markers of myogenesis [9]. High $M y o D$ expression in muscle satellite cells represses cell renewal and promotes terminal differentiation [10]. MyoD family inhibitor (MDFI) functions as a negative regulator of muscle growth by inhibiting muscle hypertrophy [11, 12]. Previous studies on I-mfa (inhibitor of MyoD family a), the mouse MDFI homolog, showed that $I-m f a$ interferes the myogenic process by masking the myogenic factor nuclear localization signal and by preventing transcription factors binding to DNA [11]. I-mfa knockout mouse showed defects in the formation of vertebrae and ribs, which suggests I- $m f a$ also involves in cartilage formation in these tissues [13]. Miyamoto et al. reported that $\beta$-catenin interacted with I-mfa and that the interaction was enhanced by Wnt3a. They also found that the interaction between $\beta$-catenin and I-mfa ameliorates the inhibition of I-mfa on myogenesis [12]. As far as we know, there is no published data on the effects of miRNA regulating MDFI expression in the muscle regeneration process.

MicroRNAs (miRNAs) is small non-coding RNA molecules (containing 22 nucleotides) found in plants, animals, and some viruses [14]. miRNAs exert their functions in RNA silencing by binding to complementary sequences of its target RNA [15, 16]_ENREF_17. For example, in mice, miR-1 and miR-133 are clustered on the same chromosomal loci and transcribed together in a tissue-specific manner during development, and then miR-133 enhances proliferation by targeting serum response factor 3' UTR, whereas miR-1 promotes myogenesis through targeting histone deacetylase 4 [17]. MiR-27b was differently expressed in the same muscle of different pig breeds, the different muscles of a pig, and the different development period of the muscle in the miRNA microarray data [18]. These results suggest miR-27b might play a critical role in pig muscle development. Crist et al. reported that miR-27b binds directly to Pax3 3'-UTR; miR-27b delayed satellite cells differentiation and down-regulated the satellite cells entry into the myogenic differentiation program [19]. MiR-27b has also been shown to repress expression of myostatin, a myogenic inhibitor, leading to double-muscled phenotype in cattle [20]. We know that MDFI is a functional gene and it inhibits myogenesis, and miR-27b promotes muscle development through targeting functional genes. However, there has been no report on whether MDFI is the target gene of miR-27b. Therefore, the objective of this study is to explore whether there is a regulatory relationship between miR-27b and MDFI during muscle development.

\section{Materials and Methods}

All animals used in this study were with the approval of the College of Animal Science, South China Agricultural University. All experiments were conducted following 'the instructive notions with respect to caring for laboratory animals' issued by the Ministry of Science and Technology of the People's Republic of China.

\section{Isolation and culture of porcine muscle satellite cells}

Three one-day-old male Landrace piglets (approximately $1.5 \mathrm{~kg}$ ) were anesthetized using sodium pentobarbital, and muscles from legs were used for PSCs isolation. The method used for the PSCs isolation has been described previously [5, 8]. Briefly, the hind deltoid muscles were dissected and rinsed with phosphate buffered saline (PBS) containing 0.5\% Penicillin-Streptomycin Solution (GIBCO) to remove blood vessels and other connective tissues. The muscle tissues were sliced into $1 \mathrm{~mm}^{3}$ pieces and digested for $2 \mathrm{~h}$ in PBS containing $0.1 \%$ collagenase II (GIBCO, Grand Island, NY, USA). Then the mixture was passed 


\section{Cellular Physiology Cell Physiol Biochem 2018;46:2271-2283 \begin{tabular}{ll|l} 
and Biochemistry Published online: Nay 07, 2018 & $\begin{array}{l}\text { @ 2018 The Author(s). Published by S. Karger AG, Basel } \\
\text { www.karger.com/cpb }\end{array}$ \\
\hline
\end{tabular}}

Hou et al.: MiR-27b Promotes Muscle Development through MDFI

through a $100 \mu \mathrm{m}$ cell strainer to remove large tissue residues. The flow-through mixture was washed three times with PBS and passed through a $70 \mu \mathrm{m}$ cell strainer. The flow-through cells were plated into $75 \mathrm{~cm}^{2}$ flasks containing DMEM/F12 medium with 20\% fetal bovine serum (FBS, GIBCO) and $0.5 \%$ chicken embryo extract. Three hours later, the unattached cells were transferred to another new flask. On the next day, cells in the flasks were treated with $0.25 \%$ trypsin for $5 \mathrm{~min}$, washed with PBS and re-plated in a new flask. After 60 to $90 \mathrm{~min}$ the upper suspension was transferred to a new flask to collect satellite cells. Cell purity was examined by PAX7 immunofluorescence assay (Fig. 9). The purified satellite cells were then plated into 6-well cell culture plate at a density of $2.5 \times 10^{4} \mathrm{cells} / \mathrm{cm}^{2}$. The PSCs were cultured in DMEM/F12 supplemented with $10 \%$ fetal bovine serum, $100 \mu \mathrm{g} / \mathrm{mL}$ streptomycin, and $100 \mathrm{U} / \mathrm{mL}$ penicillin at $37^{\circ} \mathrm{C}$ under a humidified $5 \%$ $\mathrm{CO}_{2}$ atmosphere. When the PSCs reached approximately $95 \%$ confluency, cells were allowed to differentiate in DMEM/F12 with $2 \%$ horse serum, $100 \mathrm{U} / \mathrm{mL}$ penicillin, and $100 \mu \mathrm{g} / \mathrm{ml}$ streptomycin (GIBCO BRL) for 7 days. After differentiation, the cells were washed twice with cold phosphate-buffered saline (PBS) before protein extraction or total RNA extraction.

\section{PSCs transfection}

PSCs were seeded in 6-well plates at the density of $2 \times 10^{5}$ cells per well. PSCs transfected with pCDNA3.1-MDFI, siRNA-MDFI, miR-27b inhibitor, miR-27b mimics or control by Lipofectamine 3000 (Invitrogen, Carlsbad, CA, USA), according to the manufacturer's instructions. We used qRT-PCR to detect transfection efficiency after each transfection. We transfected the PSCs with miR-27b mimic or inhibitor when the cells reach approximately $60 \%$ confluency. From previous studies conducted in our laboratory, the miR-27b mimic or inhibitor could remain active for 10-14 days. The medium was replaced with new growth medium 6 hours later, and cells were maintained in the growth medium for an additional $12 \mathrm{~h}$ before myogenic differentiation induction. MiR-27b mimics and inhibitor were purchased from GENEWIZ (Suzhou, China), and the control is the scramble sequence negative control provided by GENEWIZ.

\section{RNA extraction and PCR analysis}

Methods used for the RNA extraction and PCR analysis have been described previously [21]. In brief, the thermal cycling was performed as follows: $95^{\circ} \mathrm{C}$ for $5 \mathrm{~min}$, followed by 40 cycles of denaturation at $95^{\circ} \mathrm{C}$ for $30 \mathrm{~s}$, annealing at $58^{\circ} \mathrm{C}$ for $30 \mathrm{~s}$, and extension at $72^{\circ} \mathrm{C}$ for $30 \mathrm{~s}$. The relative expression of mRNAs and microRNA were normalized with $\beta$-actin or $U 6$ levels using the ${ }^{\Delta} \mathrm{Ct}$ method [22]. U6 is a widely used normalizer in the miRNA studies [23,24]; many previous miRNA-27b studies also used U6 as the normalizer $[25,26] .{ }^{\Delta} \mathrm{Ct}$ is defined as the ratio of the relative mRNA level of the target gene between the experimental group and the control group. Primers were designed using Primer Premier 5 according to the pig genes sequence obtained from NCBI. Primers used for PCR are shown in Table 1.

\section{Edu labeling}

The transfected PSCs were incubated in the growth medium for twenty-four hours and then were used for Edu labeling by Cell-Light ${ }^{\mathrm{TM}}$ Edu Apollo®488 In vitro Imaging Kit (RiboBio, Guangzhou, China) according to the manufacturer's instructions. The Edu labeled PSCs were washed with PBS, then observed and recorded using a Nikon TE2000-U inverted microscope (Nikon Instruments, Tokyo, Japan). The Edu positive PSCs were counted using Image Pro Plus (Media Cybernetics, Inc., Silver Spring, MD, USA).

\section{Western blot analysis}

The method used for the Western blot analysis has been described previously [21]. In brief, homogenized tissues or

Table 1. Primers Used for PCR Analyses

\begin{tabular}{lcc}
\hline Gene (Pig) & Forward sequence (5'-3') & Reverse sequence (5'-3') \\
\hline MDFI & CCTGGACAGCACTGACCTCG & TGGTACTGCCCTTGCTCTTCT \\
MyoD & TGCGTATTCTCAACCCCTTC & AGTATGCAAGGGTGGAGTGG \\
Myogenin & AGGCTACGAGCGGACTGA & GCAGGGTGCTCCTCTTCA \\
Myosin & AGACGAGGAAGAGCACG & CTCAATGTAGCGGATGG \\
MyHC & AAGGCATCATCAAGGACACTC & TGCGGCAGGTTGGCTCT \\
CCNB & CGGGATCCATGGCGCTC & CCGCTCGAGTTACACCT \\
CCND & TGTTTGCAAGCAGGACTTTG & ACGTCAGCCTCCACACTCTT \\
CCNE & TGGGCTTCAGCAGGATGATG & ACGGAACTGCTGCAGGCTGT \\
P21 & AGGACCATGTGGACCTGTTG & CGGCGTTTGGAGTGGTAGAA \\
PCNA & TTCGGTGCCTTTCTTCC & CCTTCCCTTTCGTGAGC \\
$\beta$-actin & ACGGGACATCAAGGAGAAGC & GGCACTGTGTTGGCGTAGAG \\
Gene (Mice) & Forward sequence (5'-3') & Reverse sequence (5'-3') \\
MDFI & GAGCGGTCAGTGCCCTTCT & CAGGGTGAGTGGATCTTGCTAC \\
MyoD & CGCCTGAGCAAAGTGAATGAG & GCGGTCCAGGTGCGTAGAA \\
Myogenin & CCGTGGGCATGTAAGGTG & AGGCGCTCAATGTACTGGAT \\
CCND & CGGCAGTCAAGGGAATGGT & TGAGGAGCAGAAGTGCGAAGA \\
P21 & CGAGTTCACCGTCAGCA & TCTTCAAGACAAACCCATCA \\
$\beta$-actin & TGCTGTCCCTGTATGCCTCT & CTTTGATGTCACGCACGATTT \\
\hline
\end{tabular}




\section{Cellular Physiology Cell Physiol Biochem 2018;46:2271-2283 and Biochemistry Published online: May 07, $2018 \quad \begin{aligned} & \text { DOI: 10.1159/000489595 } 2018 \text { The Author(s). Published by S. Karger AG, Basel } \\ & \text { www.karger.com/cpb }\end{aligned}$}

Hou et al.: MiR-27b Promotes Muscle Development through MDFI

cells were lysed in RIPA buffer containing protease inhibitors (1 $\mu$ M PMSF). Thirty-microgram protein lysates were separated with SDS-PAGE and then electroblotted to polyvinylidene fluoride. Electrophoresis supplies were purchased from Bio-Rad (California, USA). The membranes were blocked with $6 \%$ skim milk buffer for $2 \mathrm{~h}$ at room

Table 2. Antibody validation profile

\begin{tabular}{lcccc}
\hline Primary antibody & Clone & Company & Catalog No. & Dilution \\
\hline CCNB & Monoclonal & abcam & ab212977 & $1: 2000$ \\
CCND & Polyclonal & Bioss & bs-0623R & $1: 700$ \\
CCNE & Polyclonal & abcam & ab33911 & $1: 2000$ \\
MDFI & Monoclonal & Bioss & bs-18743R & $1: 600$ \\
MyoD & Monoclonal & abcam & ab16148 & $1: 2000$ \\
Myogenin & Monoclonal & abcam & ab1835 & $1: 2000$ \\
Myosin & Monoclonal & CST & \#8824 & $1: 3000$ \\
$\beta$-Actin & Monoclonal & Bioss & bsm-33036M & $1: 1000$ \\
Secondary antibody & Conjugate Used & Company & Catalog No. & Dilution \\
Goat Anti-mouse IgG & HRP & Bioss & bs-0296G & $1: 3000$ \\
Goat Anti-rabbit IgG & HRP & Bioss & bs-0295G & $1: 3000$ \\
\hline
\end{tabular}
temperature and incubated with different antibodies overnight at $4{ }^{\circ} \mathrm{C}$ followed by incubation with horseradish peroxidase-conjugated secondary antibodies at room temperature for $1 \mathrm{~h}$. The protein quantification was using Image J. The antibodies used in this study are listed in Table 2.

Target gene prediction

The target gene prediction was conducted using the software mirTargets 1.2 in conjunction with TargetScan, MicroCosm, Pictar and miRDB databases.

\section{Luciferase Reporter Assay}

MDFI 3' UTR sequence and MDFI-3'UTR-mut sequence were amplified and inserted into pmirGLO Vector (Ambion, Carlsbad, CA, USA), respectively. For the luciferase reporter assay, HEK 293T cells were cotransfected with pmirGLO-MDFI-3'UTR plus either miR-27b mimics or Control for 48 hours. Either pmirGLO or pmirGLO-MDFI-3'UTR-mut was used as a control for pmirGLO-MDFI-3'UTR. The activities of firefly and Renilla luciferases were determined using the Dual-Luciferase Reporter Assay System (Promega, Madison, WI, USA), and firefly luciferase activity was normalized to that of Renilla luciferase.

The MDFI 3'UTR sequence, the marked sequence is the seed sequence

The wild-type MDFI 3' UTR:

CCCCCACCCCTGGGCCC

GGGTTGGGGGGTGCCTGTCC

CAGGGGTGGGATTGGAGGAC

AGTTGGGGGGGCGGGGCAGG

CACTGCCCCTGTGCCTGCCTC

AATGTGAAAAGAGACACCCC

TGACCCGGACTGGGACCCAGA

GGGGTGGGGTGGGTGGGAACC

AAACGAACCCAACTAGTACCC

TTAAGGATCTGGCATTGCTGT

CTAGCCTGGGAACTTCCATATG

AAAAAACTGGAAGGGGAGAGGG

TAAAGATAAGTGTCCATCTCA

The mutated MDFI 3' UTR:

CCCCCACCCCTGGGCCCCC

GGGGTGCCTGTCCTCCTCGCTGG

AGGACCAGTCCTGGATCAGCTGCC

GGGGAGGCTTGGACGAGGGGCTGT

CACCAGGCTTAGAGGACAGCAAAA

CTCCCTGACCCGGACTGGGACCCAG GGGTGGGTGGGAACCCGTATAGAA ACCCACCCGTGAGGATGTGGGTTCG GTGCAGGCCGGCAGCTGCCGCTCT

ATAAAAAAAGCAAAAAAAAAAAA GTCTGCTCTACTTGTTGCTGTAAA

$$
\begin{gathered}
\text { CCCCCCACCCAGGGCCCTCCC } \\
\text { TCCTCGCTGGTTTCCAAGGAC } \\
\text { CAGTCCTGGATCAGCTGCCA } \\
\text { GACACGGGGGGAGGCTTGGA } \\
\text { CCATCACCCCCACCACCACCA } \\
\text { ACCCGACCTCAGCCAA } \\
\text { GGGCGGGGCTGGGCTTGGGCT } \\
\text { CGTATAGAAAAGACTGGAGGA } \\
\text { ACCCGTGAGGATGTGGGTTCG } \\
\text { GGCTGTGGTGCAGGCCGGCA } \\
\text { CTGCAAATGCAGCCCTATAAAA } \\
\text { AGGGAATGACTGGGGGGAGGGT }
\end{gathered}
$$

CCCCACCCAGGGCCCTCCCGGAC TTTCCAAGGACAACAGCACGGAG AGGGCTGCGGAACACTAGGAAAGT GTCTACCTCTCACTGCCCCTGTGCC TGTGAAAAGAGACACCCCACCCGAC AGGGCGGGGCTGGGCTTGGGCTCGG AAGACTGGAGGAGTTCCCATCGTGG ATCCCTGGCCTTGCTCAGTGGGTTA GATTCAACCCCTAGCCTGGGAACT AAAAAAAAAAACTGGAAGGGGAG TAAAGATAAGTGTCCATCTCA

\author{
GGACCCCAGCCCAGTGACAG \\ AACAGCACGGAGGACCTGGG \\ GGGCTGCGGAACACTGTGAA \\ CGAGGGGCTGTGTCTACCTCT \\ CCAGGCTTAGAGGACAGCAA \\ GCACCTCCCCAGGCTCCTCCC \\ CGGGACAGGGTGGGATGGGAT \\ GTTCCCATCGTGGCGCAGCGG \\ ATCCCTGGCCTTGCTCAGTGGG \\ GCTGCCGCTCTGATTCAACCC \\ AAAGCAAAAAAAAAAAAAAAAA \\ CTGCTCTACTTGTTGCTGTAAA
}

CCCAGCCCAGTGACAGGGGTTGG GACCTGGGCAGGGGTGGGATTGG TGGGGGGGCGGGGCAGGGACACGG TGCCTCCCATCACCCCCACCACCAC CTCAGCCAAGCACCTCCCCAGGCTC GACAGGGTGGGATGGGATGGGGTG CGCAGCGGAAACGAACCCAACTAGT AGGATCTGGCATTGCTGTGGCTGTG TCCATATGCTGCAAATGCAGCCCT AGGGAGGGAATGACTGGGGGGAGG 
Hou et al.: MiR-27b Promotes Muscle Development through MDFI

\section{Tissue sampling and H\&E}

Mice were purchased from Guangdong Medical Lab Animal Center. Lentivirus containing siRNA-MDFI or siRNA-Control were purchased from Shanghai JiKai Gene Chemical Technology Co., LTD. Twelve mice were divided into two groups with six mice in each group. Mice were injected with LV- siRNA-Control or LV- siRNA-MDFI. Gastrocnemius muscles were dissected from each mouse one week after injection for extracting total RNA and proteins. Mice gastrocnemius muscles were stained with Hematoxylin and Eosin (H\&E), and the cross-section area of individual myofibers was photographed using Nikon TE2000-U inverted microscope (Nikon Corporation, Tokyo, Japan) and measured using Image-Pro Plus (IPP) 6.0 software (Media Cybernetics, Inc., Silver Spring, MD, USA).

\section{Statistical analysis}

All data are expressed as the mean \pm standard error of the mean (S.E.M.), and at least three independent individuals or replicates were used per group. The assumptions of normality of data and homogeneity of variances between the groups was analyzed by SPSS. Significant differences between treatment groups were determined by one-way ANOVA (SPSS 18.0, Chicago, IL, USA). Significance was achieved when $p<0.05$. $*$ is $p<0.05$ and ${ }^{* *}$ is $p<0.01$.

\section{Results}

MiR-27b inhibits PSCs proliferation

MiR-27b mimics and inhibitor were transfected into PSCs for 36h. MiR-27b mimics reduced $(p<0.05)$ the percentage of Edu positive cells and miR-27b inhibitor increased $(p<0.05)$ the percentage of Edu positive cells (Fig. 1A, 1B). The qRT-PCR result showed miR$27 \mathrm{~b}$ mimics decreased $(p<0.05)$ mRNA level of $C C N B, C C N D, C C N E$, and PCNA and increased $(p<0.05)$ p21 mRNA level (Fig. 1C). Western blot confirmed that miR-27b mimics reduced CCNB $(p=0.062)$, CCND $(p<0.05)$, and CCNE $(p<0.05)$ protein level, and increased p21 $(p<0.01)$ protein level (Fig. 1D). Consistent with these results, after transfection with miR27b inhibitor, mRNA level of CCNB, CCND, CCNE, and PCNA were increased $(p<0.05)$ and p21 mRNA level was decreased $(p<0.01$, Fig. 1C). Western blot confirmed that miR-27b inhibitor increased CCNB $(p<0.01)$, CCND $(p<0.01)$, and CCNE $(p<0.05)$ protein level, and decreased p21 $(p<0.01)$ protein level (Fig. 1D). The relative protein levels obtained using WB bands gray scanning were presented in Fig. 8A. These results show miR-27b inhibits PSCs proliferation.

\section{MiR-27b promotes PSCs differentiation}

PSCs were transfected with miR-27b mimics or inhibitor and changed to the differentiation medium for seven days. We measured the differentiation of PSCs by the antiMyHC immunofluorescent assay. Overexpressing miR-27b increased the MyHC positive cells while inhibiting miR-27b lowered the MyHC positive cells (Fig. 2A). Overexpression of miR-27b elevated the mRNA level of MyoD ( $p<0.05)$, Myogenin $(p<0.05)$, Myosin $(p<0.05)$ and $M y H C(p<0.01)$ (Fig. 2B). Western blot result showed overexpression of miR-27b also elevated protein level of MyoD ( $p<0.05)$, Myogenin $(p<0.01)$, and Myosin $(p<0.05)$ (Fig. $2 \mathrm{C}$ ). The relative protein levels obtained using WB bands gray scanning were presented in Fig. 8B. Consistent with these results, miR-27b inhibitor decreased the myogenic-related gene expression (Fig. 2B, 2C). These results show miR-27b promotes PSCs differentiation.

\section{MDFI is a direct target gene of miR-27b}

To further investigate the potential mechanism by which miR-27b regulates the PSCs myogenesis, we need to identify the target gene of miR-27b. Through the sequence alignment we found that the miR-27b seed sequence is highly conserved (Fig. 3A), the miR$27 \mathrm{~b}$ complementary seed sequence in the MDFI 3'UTR is also highly conserved (Fig. 3B), so there may be a potential binding site in MDFI UTR for miR-27b (Fig. 3C).

To verify the targeted relationship between miR-27b and $M D F I$, we first measured the expression of MDFI through qRT-PCR and Western blot in the PSCs transfected with miR-27b 
Fig. 1. MiR-27b inhibits PSCs proliferation. A. MiR-27b mimics lowered the percentage of Edu positive PSCs, and miR-27b inhibitor increased the percentage of Edu positive PSCs. Representative images of the immunofluorescent staining for proliferating PSCs are shown. Proliferating PSCs were labeled with Edu fluorescent dye (red). B. The quantitative data of proliferating PSCs in Fig. A. C. qRT-PCR confirmed miR-27b is negatively correlated with proliferation-related genes in PSCs transfected with miR-27b mimics and inhibitor. D. Western blot result showed the protein level corresponds to the

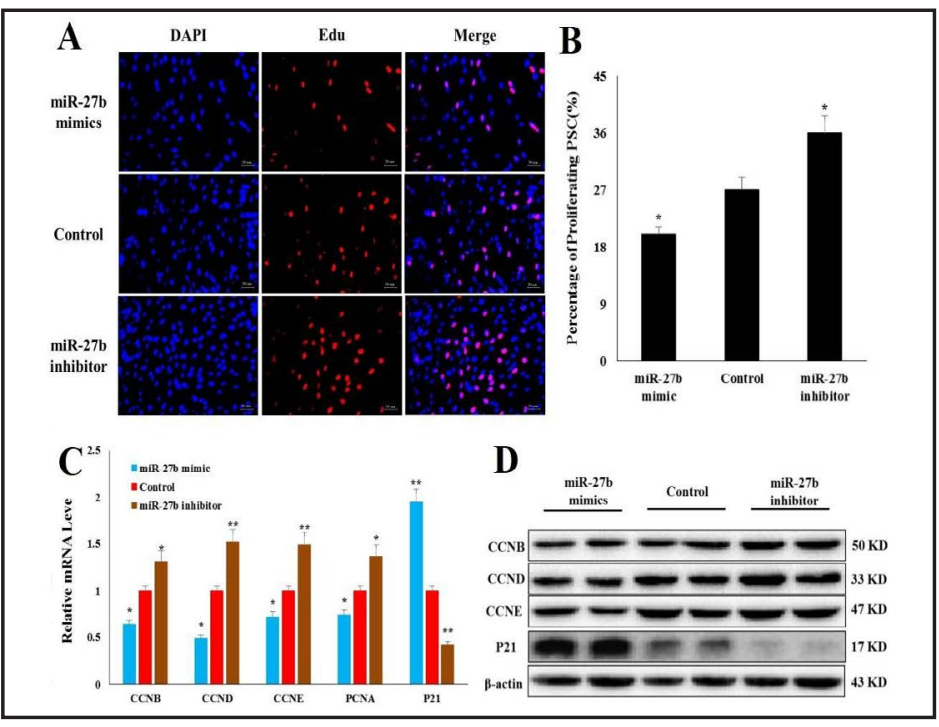
mRNA result. *, $\mathrm{p}<0.05$; ${ }^{* *}, \mathrm{p}<0.01$. The results are presented as Mean \pm S.E.M. of three replicates for each group. Magnification 200×. The scale bar on the photomicrographs represents $50 \mu \mathrm{m}$.

Fig. 2. MiR-27b promotes PSCs differentiation. We measured the expression of myogenic marker genes after PSCs were transfected with miR-27b mimics or inhibitor. A. MiR-27b mimics increased the percentage of MyHC positive PSCs, and miR-27b inhibitor decreased the percentage of MyHC positive PSCs. Representative images of the immunofluorescent staining for differentiated PSCs are shown. MyHC: red, a molecular marker of

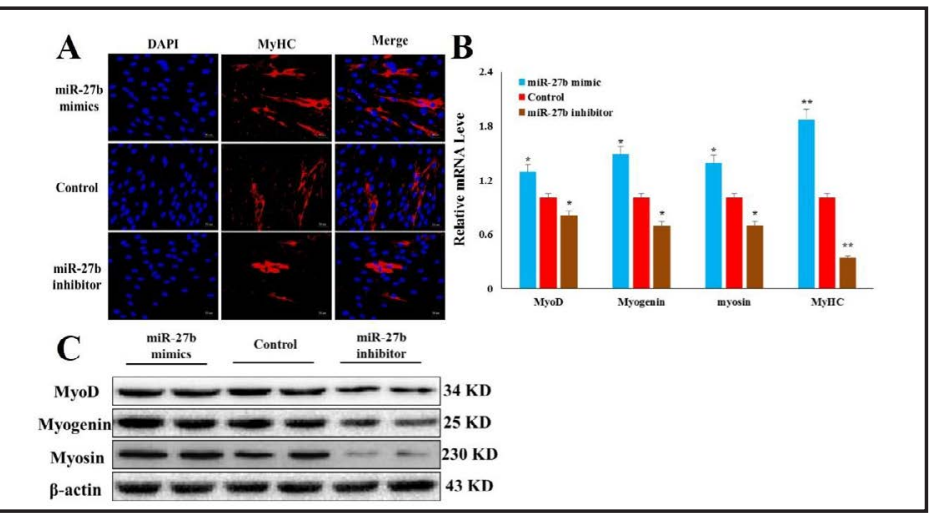
myogenesis; DAPI: blue, cell nuclei; Merge: the PSCs fused into primary myotubes are shown in the insets. B. The qRT-PCR confirmed miR-27b increased $(p<0.05)$ mRNA level of myogenic marker genes after differentiation induction. C. We used western blot to measure protein level of myogenic marker genes after differentiation induction. Western blot showed the protein result corresponds to the mRNA result after differentiation induction. *, $\mathrm{p}<0.05 ;{ }^{* *}, \mathrm{p}<0.01$. The results are presented as Mean \pm S.E.M. of three replicates for each group. Magnification 200x. The scale bar on the photomicrographs represents $50 \mu \mathrm{m}$.

mimics or inhibitor. The results showed overexpressing miR-27b decreased MDFI expression while inhibiting miR-27b increased the MDFI expression (Fig. 3D and 3E). The MDFI protein levels obtained using WB bands gray scanning were presented in Fig. 8C. Subsequently, we constructed the pmirGLO-MDFI 3'UTR recombinant vector containing the binding site of miR-27b. We found relative luciferase activity was decreased ( $p<0.01$; Fig. 3F) when HEK293T cells were co-transfected with miR-27b mimics and pmirGLO-MDFI-3'UTR. However, miR-27b mimics does not affect mutated pmirGLO-MDFI-3'UTR relative luciferase activity (Fig. 3F). This result indicates that MDFI is the direct target gene of miR-27b.

\section{MDFI promotes PSCs proliferation}

Since MDFI is the direct target gene of miR-27b, we then explored the role of MDFI in PSCs development. We overexpressed and inhibited MDFI expression in transfected PSCs with pcDNA3.1(+)-MDFI recombinant vector and siRNA-MDFI, respectively. Overexpressing $M D F I$ 
Hou et al.: MiR-27b Promotes Muscle Development through MDFI

Fig. 3. MDFI is a direct target gene of miR-27b. A. The sequence of mature miR-27b of different species. B. The alignment result of MDFI 3'UTR sequence from different species. Red: the complete complementary sequence of the MDFI 3'UTR. C. The complementary pairing of ssc-miR-27b with the targeted gene MDFI 3'UTR. D. MDFI protein level was negatively correlated with miR-27b level. E. qRT-PCR result confirmed MDFI mRNA level was negatively correlated with miR-27b level. F. Luciferase reporters were transfected into

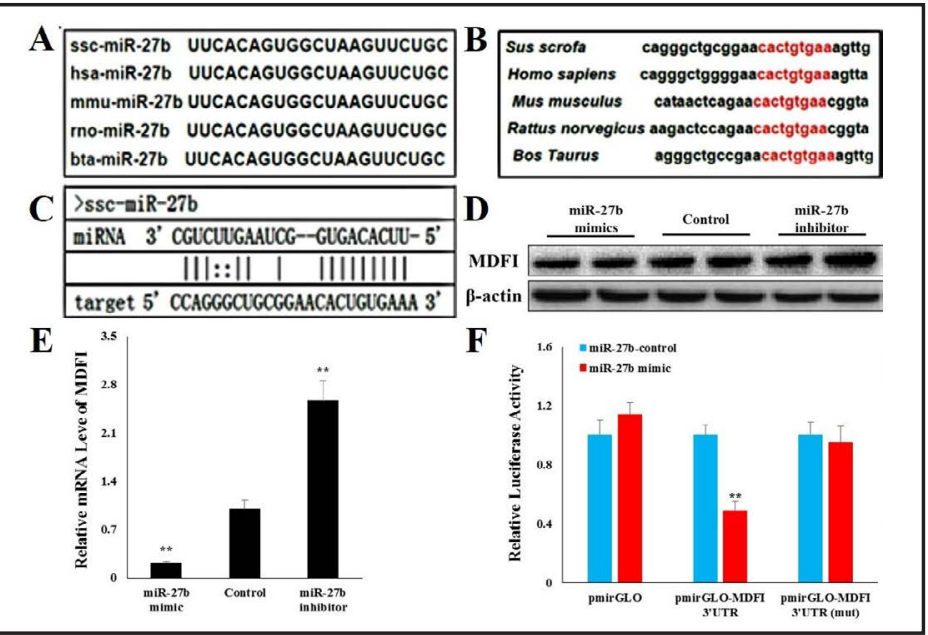
HEK-293T cells with either miR-

27b mimics or control, respectively. Luciferase activity was determined $24 \mathrm{~h}$ after transfection. $* *, \mathrm{p}<0.01$. The results are presented as Mean \pm S.E.M. of three replicates for each group.

increased $(p<0.01)$ the percentage of Edu positive cells and siRNA-MDFI decreased $(p<0.05)$ the percentage of Edu positive cells (Fig. 4A, 4B). The qRT-PCR result showed pcDNA3.1(+)MDFI recombinant vector increased the mRNA level of CCNB $(p<0.05), C C N D(p<0.01), C C N E$ $(p<0.01)$ and PCNA $(\mathrm{p}<0.01)$ and decreased $(p<0.05) p 21$ mRNA level (Fig. 4C). Western blot result confirmed that pcDNA3.1(+)-MDFI recombinant vector increased protein level of CCNB $(p<0.05), \operatorname{CNND}(p<0.05)$, and CCNE $(p<0.05)$, and decreased $p 21(p<0.01)$ protein level (Fig. 4D). After PSCs were transfected with siRNA-MDFI, we found siRNA-MDFI decreased $(p<0.05)$ the mRNA level of $C C N B, C C N D, C C N E$, and PCNA and increased $(p<0.01) p 21 \mathrm{mRNA}$ level (Fig. 4C). Western blot result confirmed that siRNA -MDFI decreased protein level of CCNB $(p<0.05)$, CCND $(p=0.062)$, and CCNE $(p<0.05)$ and increased p21 $(p<0.01)$ protein level (Fig. 4D). The relative protein levels obtained using WB bands gray scanning were presented in Fig. 8D. From the MDFI overexpression and inhibition results, we conclude that MDFI promotes PSCs proliferation.

\section{MDFI inhibits PSCs differentiation}

We then determined the role of MDFI in PSCs differentiation. PSCs were transfected with either pcDNA3.1(+)-MDFI recombinant vector or siRNA-MDFI and then cultured in the differentiation medium for seven days. We used the anti-MyHC immunofluorescent assay to measure the differentiation of PSCs. Overexpressing MDFI decreased the MyHC positive cells while inhibiting MDFI increased the MyHC positive cells (Fig. 5A). Then the expression of $M y o D$, Myogenin, Myosin, and $M y H C$ was measured. Overexpression of MDFI lowered the mRNA level of MyoD $(p<0.05)$, Myogenin $(p<0.05)$, Myosin $(p<0.05)$ and MyHC $(p<0.01)$ (Fig. 5B). Western blot result showed overexpression of MDFI lowered the protein level of MyoD ( $p<0.01)$, Myogenin $(p<0.05)$, and Myosin $(p<0.01)$ (Fig. 5C). The relative protein levels obtained using WB bands gray scanning were presented in Fig. 8E. Consistent with these results, inhibiting MDFI increased the myogenic-related gene expression (Fig. 2B, 2C). These results show MDFI inhibits PSCs differentiation.

\section{MiR-27b promotes PSCs myogenesis by targeting MDFI.}

Since miR-27b inhibits PSCs proliferation, promotes PSCs differentiation and MDFI is a direct target gene of miR-27b, we co-overexpressed miR-27b and MDFI to verify whether MDFI attenuated the miR-27b effect on PSCs development. Through the Edu immunofluorescent staining, we found $M D F I$ weakens the miR-27b inhibition effect on PSCs proliferation. The MyHC immunofluorescent staining results shown MDFI reduced the miR-27b promotion 
Fig. 4. MDFI promotes PSCs proliferation. A. Overexpressing MDFI increased the percentage of Edu positive PSCs and interfering MDFI decreased the percentage of Edu positive PSCs. Representative images of the immunofluorescent staining for proliferating PSCs are shown. Proliferating PSCs were labeled with Edu fluorescent dye (red). B. The quantitative data of proliferating PSCs number in Fig. A. C. qRT-PCR confirmed MDFI is positively correlated with proliferation-related genes in PSCs transfected with pcDNA3.1(+)-MDFI recombinant vector and siRNA-MDFI respectively. D. Western blot result

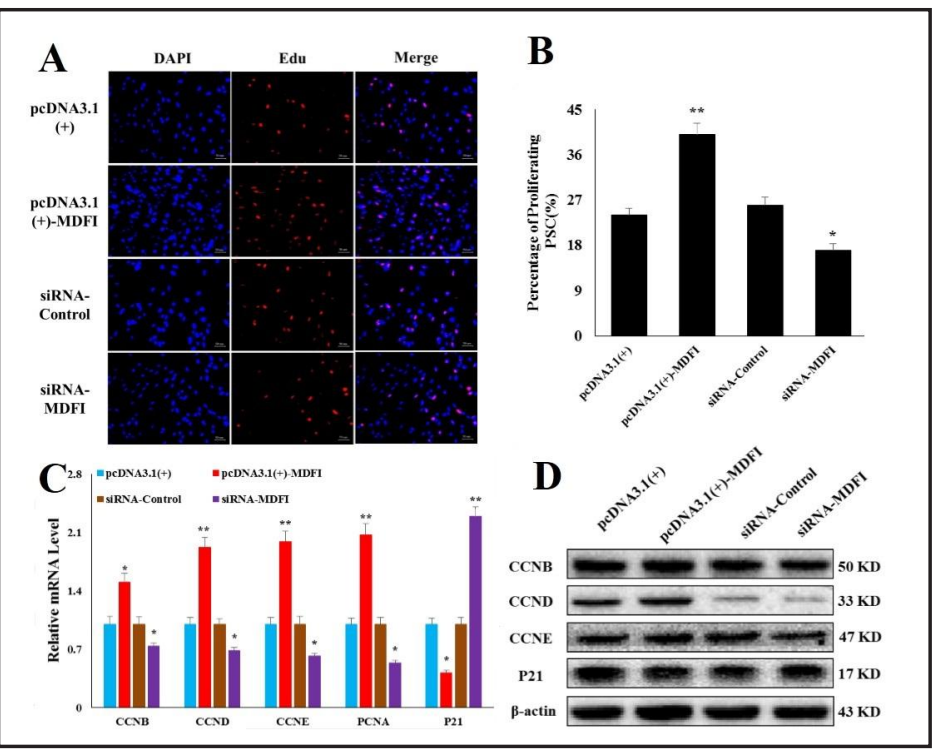
showed the protein level corresponds to the mRNA result. ${ }^{*}, \mathrm{p}<0.05 ;{ }^{* *}, \mathrm{p}<0.01$. The results are presented as Mean \pm S.E.M. of three replicates for each group. Magnification 200x. The scale bar on the photomicrographs represents $50 \mu \mathrm{m}$.

Fig. 5. MDFI inhibits PSCs differentiation. A. Overexpressing MDFI decreased the percentage of MyHC positive PSCs and interfering MDFI increased the percentage of Edu positive PSCs. Representative images of the immunofluorescent staining for differentiated PSCs are shown. MyHC: red, a molecular marker of myogenesis; DAPI: blue, cell nuclei; Merge: the PSCs fused into primary myotubes are shown in the insets. B. qRT-PCR result confirmed MDFI decreased the mRNA level of myogenic marker

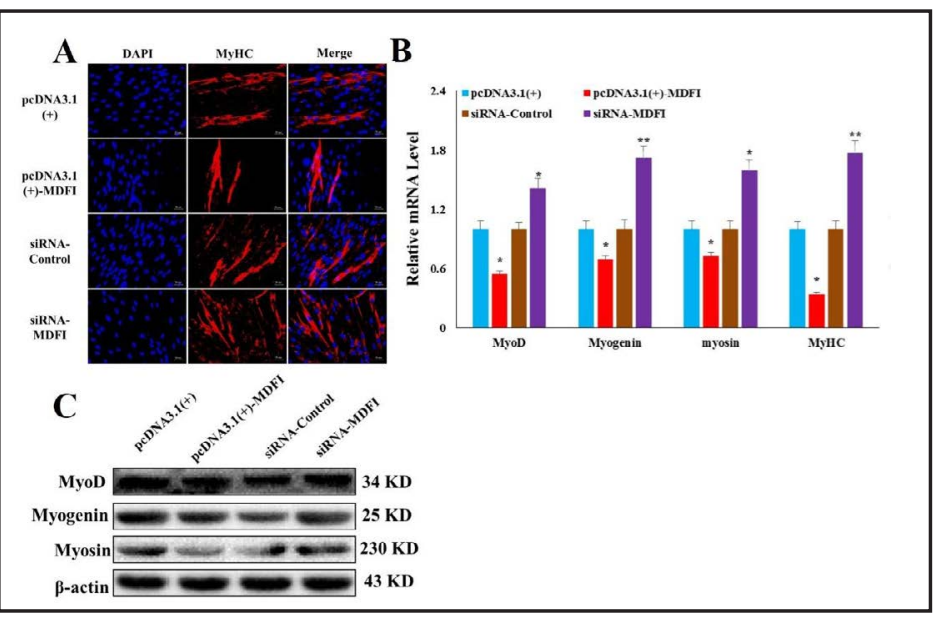
genes after differentiation induction. C. We used western blot to measure the protein level of myogenic marker genes after differentiation induction. Western blot result showed the protein level corresponds to the mRNA result after differentiation induction. ${ }^{*}, \mathrm{p}<0.05$; ${ }^{* *}, \mathrm{p}<0.01$. The results are presented as Mean \pm S.E.M. of three replicates for each group. Magnification 200×. The scale bar on the photomicrographs represents $50 \mu \mathrm{m}$.

effect on PSCs differentiation (Fig. 6A, 6B), so we conclude that miR-27b promotes PSCs myogenesis by targeting $M D F I$.

\section{Interfering MDFI promotes the muscle development in vivo}

The miRNA is known to exert their function through inhibiting the expression of their target genes. Results from our in vitro study have identified MDFI is the target gene of miR$27 \mathrm{~b}$. To our knowledge, there is no published report about the effects of MDFI on skeleton muscle regeneration after injury. Therefore, we decided to explore the role of MDFI in PSCs development and muscle regeneration after injury. We first damaged the gastrocnemius muscle of Kunming mouse by cardiotoxin (CTX) injection. Then we infected the muscle 
Fig. 6. MiR-27b promotes PSCs myogenesis by targeting MDFI. A. MiR-27b function on PSCs proliferation and differentiation requires MDFI. Representative images of the immunofluorescent staining for proliferating PSCs and differentiated PSCs are shown. B. The

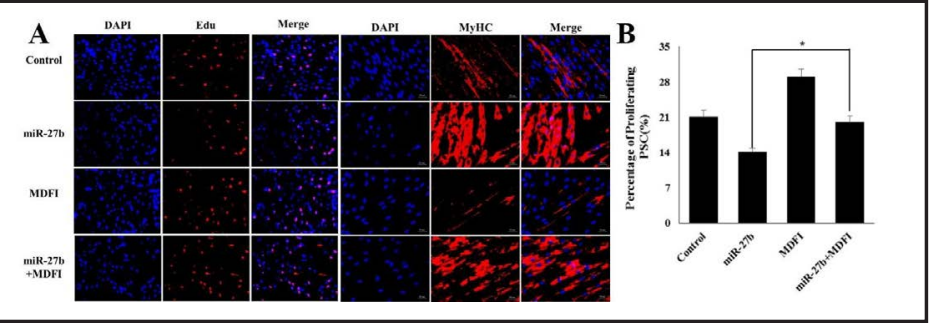
quantitative data of proliferating PSCs number in Fig. A. ${ }^{*}, \mathrm{p}<0.05$. The results are presented as Mean \pm S.E.M. of three replicates for each group. Magnification 200×. The scale bar on the photomicrographs represents $50 \mu \mathrm{m}$.

Fig. 7. Interfering MDFI promotes the muscle development in vivo. A. H\&E staining of hind limb muscle one week after injection with lentivirus packaged siRNA (LV-Si-MDFI). B. Total fibers number in one field after interfering hind limb muscle MDFI. C. The average area of muscle fiber cross-sections was increased after interfering MDFI. D. The average diameter of myofiber was increased after interfering MDFI.E. qRT-PCR detection of
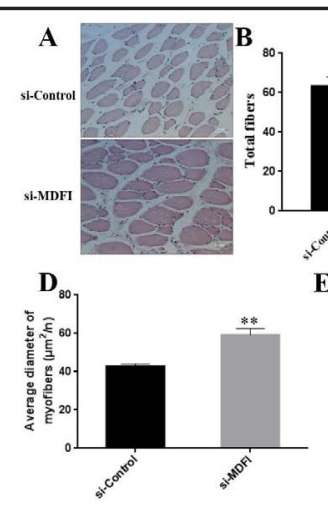

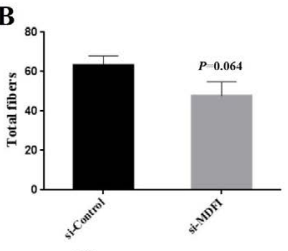

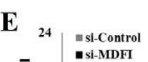

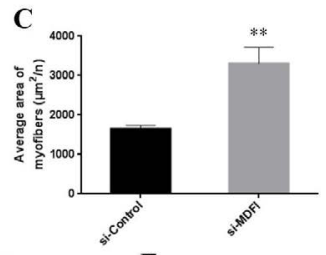
F

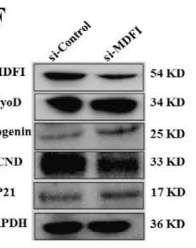
MDFI, the mRNA level of myogenicrelated genes. F. Western blot determination of the protein level of myogenic-related genes. *, p<0.05; **, $\mathrm{p}<0.01$. The results are presented as Mean \pm S.E.M. of three replicates for each group. Magnification $20 \times$. The scale bar on the photomicrographs represents $50 \mu \mathrm{m}$.

with the lentivirus packaged siRNA LV-Si-MDFI to elucidate the MDFI effect on muscle regeneration. We harvested the gastrocnemius muscles one week after the injection and measured the morphological characteristics of gastrocnemius muscles and the expression of related genes. Inhibition of $M D F I$ increased the diameter of single muscle fibers (Fig. 7A, 7C, 7D). But the total number of fibers in one field was no difference between the two groups (Fig. 7B). Inhibiting MDFI increased ( $p<0.01)$ the mRNA level of $M y o D$ and Myogenin, decreased the mRNA level of MDFI $(p<0.01)$ and CCND1 ( $p<0.05)$, Fig. 7E). Western blot result showed interfering MDFI lowered the protein level of MDFI $(p<0.01)$ and CCND $(p<0.01)$, increased the protein level of MyoD ( $p<0.05)$, and P21 ( $p<0.01)$ (Fig. 7F). The relative protein levels obtained using WB bands gray scanning were presented in Fig. $8 \mathrm{~F}$. These results demonstrate that inhibition of MDFI in vivo promoted the muscle regeneration after injury.

\section{Discussion}

Skeletal muscle comprises $45 \%$ of the human body mass, and musculoskeletal system injuries are common [27]. Muscle injuries in sports account for $10 \%-55 \%$ of all muscle injuries. Over $90 \%$ of these injuries are either contusions or strains [28, 29].. Traumatic injuries from motor vehicle accidents contribute to an increasing need for better treatment options for muscle regeneration [30]. After an injury, muscle satellite cells are activated, re-enter the cell cycle to proliferate and subsequently differentiate into myoblasts. The proliferation and differentiation of muscle satellite cells is the basis of muscle regeneration $[31,32]$. Therefore, understanding of the mechanisms associated with muscle development is essential for devising the alternative treatments for muscle regeneration. 
The development of muscle satellite cells is affected by many factors, including myogenic regulatory factors (such as MyoD, Myf5, Myogenin and MRF4), miRNAs, the muscle satellite cells microenvironment and age [33, 34]. Pig is a useful medical model for humans due to its similarity in size and physiology. In our study, we used pig muscle satellite cell as the model to explore the regulatory relationship between the MDFI and miR$27 \mathrm{~b}$ in vitro. Since in vitro study may not represent the true physiological state in living organisms, we also conducted the MDFI in animals. The muscle regeneration model after Cardiotoxin damage has been used in mice [35, 36], we chose to conduct the in vivo study in mice. Since our laboratory has explored the effects of different concentrations of CTX on the degree of muscle damage in Kunming mice and has established a complete CTX damaging the mouse muscle protocol we chose this particular model for this study.

For any experiment, a

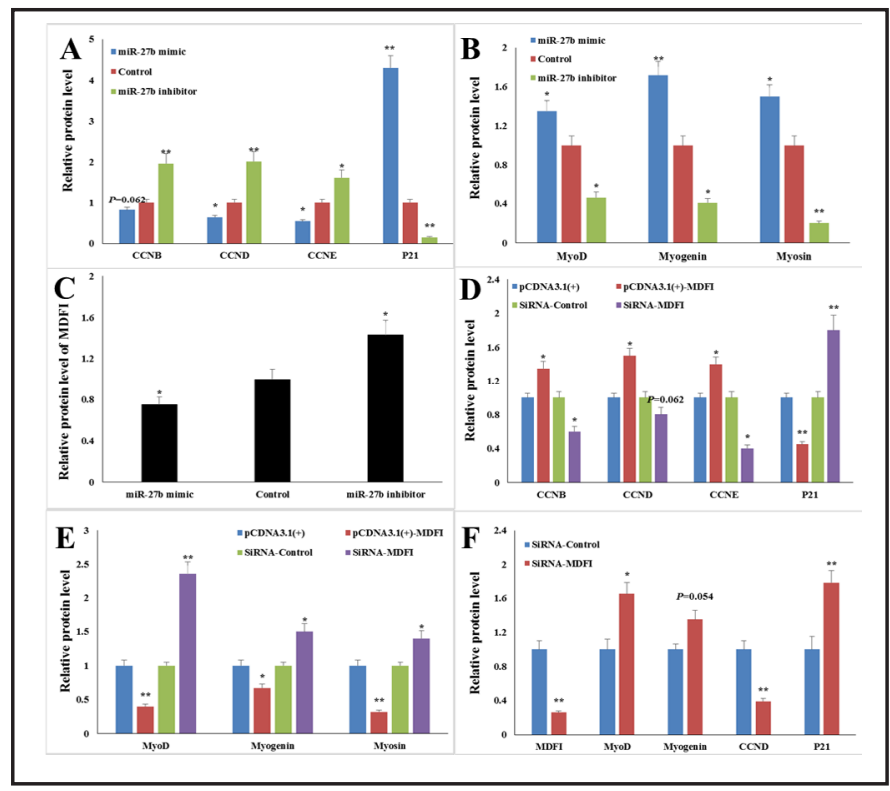

Fig. 8. WB bands gray scanning quantitative results. A for Figure 1D; B for Figure 2C; C for Figure 3D; D for Figure 4D; E for Figure 5C; $\mathrm{F}$ for Figure 7F.

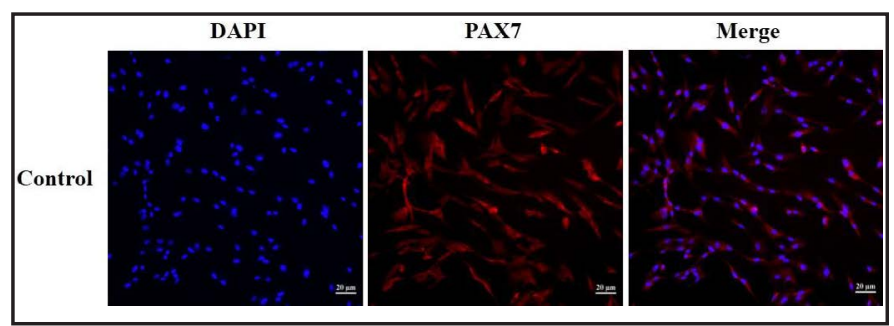

Fig. 9. The isolated satellite cells were identified by PAX7 immunofluorescence assay. negative control should be included to allow for control of parameters such as intra-individual variability, variability among animal strains, tools utilized. Since previous research conducted by our laboratory on miR-34c has shown the LV-Si-Control does not affect the muscle development [37], we did not include a negative control in this experiment. However, the difference between si-MDFI and control mice was significant, and there were six mice in each group, so we believe this result should still be valid.

$M y o D$, a myogenic transcription factor, has been extensively studied and it plays an essential role in muscle development. However, $M D F I$, the inhibitor of $M y o D$, we can find only three $M D F I$-related papers. The role of $M D F I$ in regulating muscle development remains to be elucidated.

After activation, muscle satellite cells re-enter the cell cycle to proliferate. After several rounds of replication, muscle satellite cells exit the cell cycle and differentiate into myoblasts. Finally, the myoblasts fuse to the existing muscles. P21 and cyclins are the key factors regulating the cell cycle. Since $C C N D$ is the first cyclin produced in the cell cycle, we measured CCND expression as the PSCs proliferation marker. CCND binds to the existing cyclindependent kinases (CDK) 4, forming the active CCND-CDK4 complex. Then, CCND-CDK4, in turn, phosphorylates the retinoblastoma susceptibility protein $(\mathrm{Rb})$. $\mathrm{Rb}$ dissociates from the E2F/DP1/Rb complex, activating E2F. E2F promotes expression of various genes such as CCNE and DNA polymerase. Therefore, we also measured CCNE expression as the PSCs 
Hou et al.: MiR-27b Promotes Muscle Development through MDFI

proliferation marker. CCNE binds to CDK2, forming the CCNE-CDK2 complex, which pushes the cell from $G_{1}$ to $S$ phase. CCNB-CDK1 complex activation was causes breakdown and prophase initiation. Subsequently, CCNB-CDK1 deactivation causes the cell to exit mitosis $[6,38,39]$. The P21 protein is a cell cycle regulator, and it binds to cyclin-CDKs complexes and inhibits cyclin-CDKs activities [7, 40]. Thus, CCNB and P21 have also been used as the PSCs proliferation markers in our study. MyoD is also involved in removing cells from the cell cycle through increasing the $\mathrm{p} 21$ transcription. $M y o D$ is inhibited by CDKs, CDKs, in turn, are inhibited by p21. Thus MyoD enhances its own activity in a feed-forward manner [41, 42]. In our study, through the mRNA, protein and cell number results, we established that $M y o D$ expression is increased and p21 expression is decreased by interfering $M D F I$, and finally, resulted in elevated PSCs proliferation. MDFI can identify the basic helix loop helix (bHLH) structure on the MyoD $[11,13]$. Since many myogenic transcription factors contain the bHLH structure, future studies will include the identification of additional transcription factors regulated by the MDFI.

The miRNAs are critical regulators of many biological processes through modulating expression of genes in the post-transcriptional level. Proliferation and differentiation are mutually exclusive during muscle regeneration, and miRNAs are critically involved in balancing these two processes [43]. Many miRNAs, such as miR-1, miR-133, miR-29, miR214, miR-206, miR-486, miR-208b, and miR-499 were involved in the regulation of skeletal myogenesis by binding to its target genes $[44,45]$. For example, in mice, miR-1 and miR133 are clustered on the same chromosomal loci and transcribed together in a tissuespecific manner during development, but miR-133 enhances proliferation by repressing serum response factor, whereas miR-1 promotes myogenesis through repressing histone deacetylase 4 [17]. Additionally, our previous study found miR-34c form a reciprocal regulatory loop with its target gene Notch 1[37]. These reports support the claim miRNAs are critically involved in muscle regeneration.

miR-27b has been shown to inhibit cardiac cell growth and induce cardiac hypertrophy by targeting PPAR [46]. MiR-27b interference was reported to increase Pax3 expression leading to muscle stem cell proliferation, delaying the onset of differentiation; miR-27b mimics ensures rapid and robust entry into the myogenic differentiation program [19]. In our study, through the mRNA, protein and cell count results, we ascertained that miR-27b decreased MDFI expression, which leads to inhibition of PSCs proliferation and promotion of PSCs differentiation. In conclusion, our study demonstrated that miR-27b inhibits PSCs proliferation and promotes PSCs differentiation in vitro. The mechanism is miR-27b inhibits the expression of its target gene, MDFI. Through interfering MDFI expression in vivo, our results showed that inhibiting $M D F I$ expression promotes mice muscle regeneration after injury. These results expand our understanding of muscle development mechanism in which miRNAs and genes participate in controls skeletal muscle development. Furthermore, this finding has implications for obtaining the alternative treatments for muscle regeneration after injury in the future.

\section{Acknowledgements}

This work was supported by the National High Technology Research and Development Program 863 (\#2013AA102502); the National Natural Science Foundation of China (\#31372283); the Team Project of Guangdong Agricultural Department (2017LM2148) and the Guangdong Natural Science Foundation (2014A030310068). C.H. is supported by the USDA National Institute of Food and Agriculture, Hatch project HAW-H2037, managed by the College of Tropical Agriculture and Human Resources, University of Hawaii at Manoa.

All authors made contributions to this study. C. W., J. D., T. G. and L. H. designed the research. L. H., Y. J. and J. D. performed the research and developed the methods. H. L., J. X., H. L. and Z. P. analyzed the data. L. H., C. W. and C. H. wrote the paper. All authors read and approved the final manuscript.

\section{KARGER}




\section{Cellular Physiology Cell Physiol Biochem 2018;46:2271-2283 \begin{tabular}{l|l} 
DOI: $10.1159 / 000489595$ \\
and Biochemistry Published online: May 07, 2018 & $\begin{array}{l}\text { O 2018 The Author(s). Published by S. Karger AG, Basel } \\
\text { www.karger.com/cpb }\end{array}$
\end{tabular}}

Hou et al.: MiR-27b Promotes Muscle Development through MDFI

\section{Disclosure Statement}

The authors declare to have no conflict of interests.

\section{References}

$>1$ Turner NJ, Badylak SF: Regeneration of skeletal muscle. Cell Tissue Res 2012;347:759-774.

-2 Tidball JG: Mechanisms of Muscle Injury, Repair, and Regeneration. Compr Psychiatry 2011;1:2029-2062.

- Montarras D, Morgan J, Collins C, Relaix F, Zaffran S, Cumano A, Partridge T, Buckingham M: Direct isolation of satellite cells for skeletal muscle regeneration. Science 2005;309:2064-2067.

4 Kuang S, Kuroda K, Le Grand F, Rudnicki MA: Asymmetric self-renewal and commitment of satellite stem cells in muscle. Cell 2007;129:999-1010.

5 Qin L, Xu J, Wu Z, Zhang Z, Li J, Wang C, Long Q: Notch1-mediated signaling regulates proliferation of porcine satellite cells (PSCs). Cell Signal 2013;25:561-569.

6 Gopinathan L, Ratnacaram CK, Kaldis P: Established and novel Cdk/cyclin complexes regulating the cell cycle and development. Results Probl Cell Differ 2011;53:365-389.

7 Besson A, Dowdy SF, Roberts JM: CDK inhibitors: cell cycle regulators and beyond. Dev Cell 2008;14:159169.

-8 Qin LL, Li XK, Xu J, Mo DL, Tong X, Pan ZC, Li JQ Chen YS, Zhang Z, Wang C, Long QM: Mechano growth factor (MGF) promotes proliferation and inhibits differentiation of porcine satellite cells (PSCs) by downregulation of key myogenic transcriptional factors. Mol Cell Biochem 2012;370:221-230.

>9 Zhang F, Deng B, Wen J, Chen K, Liu W, Ye S, Huang H, Jiang S, Xiong Y: PPARgamma and MyoD are differentially regulated by myostatin in adipose-derived stem cells and muscle satellite cells. Biochem Biophys Res Commun 2015;458:375-380.

10 Conerly ML, Yao Z, Zhong JW, Groudine M, Tapscott SJ: Distinct Activities of Myf5 and MyoD Indicate Separate Roles in Skeletal Muscle Lineage Specification and Differentiation. Dev Cell 2016;36:375-385.

-11 Chen CM, Kraut N, Groudine M, Weintraub H: I-mf, a novel myogenic repressor, interacts with members of the MyoD family. Cell 1996;86:731-741.

12 Miyamoto K, Koseki M, Kawakami Y, Hatanaka N, Kamiike W, Ushijima T, Taniyama K: MDFI (MyoD family inhibitor), which regulates myogenic and Wnt/beta-catenin signaling pathways, is inactivated in human breast cancer by epigenetic gene silencing. Cancer Res 2007;67:1056-1056.

13 Kraut N: The gene encoding I-mf (Mdfi) maps to human chromosome 6p21 and mouse chromosome 17 Mamm Genome 1997;8:618-619.

14 Ambros V: The functions of animal microRNAs. Nature 2004;431:350-355.

15 Baek D, Villen J, Shin C, Camargo FD, Gygi SP, Bartel DP: The impact of microRNAs on protein output. Nature 2008;455:64-71.

16 He L, Hannon GJ: MicroRNAs: small RNAs with a big role in gene regulation. Nat Rev Genet 2004;5:522-531.

17 Chen JF, Mandel EM, Thomson JM, Wu Q Callis TE, Hammond SM, Conlon FL, Wang DZ: The role of microRNA-1 and microRNA-133 in skeletal muscle proliferation and differentiation. Nat Genet 2006;38:228-233.

18 McDaneld TG, Smith TPL, Doumit ME, Miles JR, Coutinho LL, Sonstegard TS, Matukumalli LK, Nonneman DJ, Wiedmann RT: MicroRNA transcriptome profiles during swine skeletal muscle development. Bmc Genomics 2009;10:

19 Crist CG, Montarras D, Pallafacchina G, Rocancourt D, Cumano A, Conway SJ, Buckingham M: Muscle stem cell behavior is modified by microRNA-27 regulation of Pax3 expression. Proc Natl Acad Sci U S A 2009;106:13383-13387.

20 Allen DL, Loh AS: Posttranscriptional mechanisms involving microRNA-27a and b contribute to fastspecific and glucocorticoid-mediated myostatin expression in skeletal muscle. Am J Physiol Cell Physiol 2011;300:C124-C137.

21 Hou L, Shi J, Cao L, Xu G, Hu C, Wang C: Pig has no uncoupling protein 1. Biochem Biophys Res Commun 2017;487:795-800. 


\section{Cellular Physiology Cell Physiol Biochem 2018;46:2271-2283

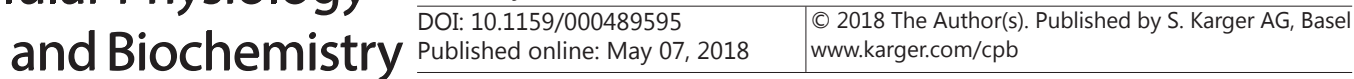

Hou et al.: MiR-27b Promotes Muscle Development through MDFI

22 Shu G, Lu NS, Zhu XT, Xu Y, Du MQ Xie QP, Zhu CJ, Xu Q, Wang SB, Wang LN, Gao P, Xi QY, Zhang YL, Jiang QY: Phloretin promotes adipocyte differentiation in vitro and improves glucose homeostasis in vivo. J Nutr Biochem 2014;25:1296-1308.

23 Huang ZP, Chen J, Seok HY, Zhang Z, Kataoka M, Hu X, Wang DZ: MicroRNA-22 regulates cardiac hypertrophy and remodeling in response to stress. Circ Res 2013;112:1234-1243.

-24 Zhou B, Wang S, Mayr C, Bartel DP, Lodish HF: miR-150, a microRNA expressed in mature B and T cells, blocks early B cell development when expressed prematurely. Proc Natl Acad Sci U S A 2007;104:70807085.

25 Shen L, Chen L, Zhang S, Du J, Bai L, Zhang Y, Jiang Y, Li X, Wang J, Zhu L: MicroRNA-27b Regulates Mitochondria Biogenesis in Myocytes. PLoS One 2016;11:e0148532.

26 Zeng X, Huang C, Senavirathna L, Wang P, Liu L: miR-27b inhibits fibroblast activation via targeting TGFbeta signaling pathway. BMC Cell Biol 2017;18:9.

27 Huard J, Yokoyama T, Pruchnic R, Qu Z, Li Y, Lee JY, Somogyi GT, de Groat WC, Chancellor MB: Musclederived cell-mediated ex vivo gene therapy for urological dysfunction. Gene Ther 2002;9:1617-1626.

28 Counsel P, Breidahl W: Muscle injuries of the lower leg. Semin Musculoskelet Radiol 2010;14:162-175.

29 Lehto MU, Jarvinen MJ: Muscle injuries, their healing process and treatment. Ann Chir Gynaecol 1991;80:102-108.

-30 Munoz-Canoves P, Carvajal JJ, Lopez de Munain A, Izeta A: Editorial: Role of Stem Cells in Skeletal Muscle Development, Regeneration, Repair, Aging, and Disease. Front Aging Neurosci 2016;8:95.

-31 Rhoads RP, Fernyhough ME, Liu X, McFarland DC, Velleman SG, Hausman GJ, Dodson MV: Extrinsic regulation of domestic animal-derived myogenic satellite cells II. Domest Anim Endocrinol 2009;36:111126.

-32 Abou-Khalil R, Yang F, Lieu S, Julien A, Perry J, Pereira C, Relaix F, Miclau T, Marcucio R, Colnot C: Role of muscle stem cells during skeletal regeneration. Stem Cells 2015;33:1501-1511.

-33 Wilschut KJ, Haagsman HP, Roelen BA: Extracellular matrix components direct porcine muscle stem cell behavior. Exp Cell Res 2010;316:341-352.

-34 Mesires NT, Doumit ME: Satellite cell proliferation and differentiation during postnatal growth of porcine skeletal muscle. Am J Physiol Cell Physiol 2002;282:C899-906.

35 Guardiola O, Andolfi G, Tirone M, Iavarone F, Brunelli S, Minchiotti G: Induction of Acute Skeletal Muscle Regeneration by Cardiotoxin Injection. J Vis Exp 2017;10.3791/54515

36 Garry GA, Antony ML, Garry DJ: Cardiotoxin Induced Injury and Skeletal Muscle Regeneration. Methods Mol Biol 2016;1460:61-71.

37 Hou L, Xu J, Li H, Ou J, Jiao Y, Hu C, Wang C: MiR-34c represses muscle development by forming a regulatory loop with Notch1. Sci Rep 2017;7:9346.

-38 Bloom J, Cross FR: Multiple levels of cyclin specificity in cell-cycle control. Nat Rev Mol Cell Biol 2007;8:149-160.

39 Hochegger H, Takeda S, Hunt T: Cyclin-dependent kinases and cell-cycle transitions: does one fit all? Nat Rev Mol Cell Biol 2008;9:910-916.

-40 Srivastava RK, Chen Q Siddiqui I, Sarva K, Shankar S: Linkage of curcumin-induced cell cycle arrest and apoptosis by cyclin-dependent kinase inhibitor p21(/WAF1/CIP1). Cell Cycle 2007;6:2953-2961.

-41 Shi L, Zhao G, Qiu D, Godfrey WR, Vogel H, Rando TA, Hu H, Kao PN: NF90 regulates cell cycle exit and terminal myogenic differentiation by direct binding to the 3'-untranslated region of MyoD and p21WAF1/ CIP1 mRNAs. J Biol Chem 2005;280:18981-18989.

42 Ishido M, Kami K, Masuhara M: Localization of MyoD, myogenin and cell cycle regulatory factors in hypertrophying rat skeletal muscles. Acta Physiol Scand 2004;180:281-289.

43 Kovanda A, Rezen T, Rogelj B: MicroRNA in skeletal muscle development, growth, atrophy, and disease. Wiley Interdiscip Rev RNA 2014;5:509-525.

44 Ge Y, Chen J: MicroRNAs in skeletal myogenesis. Cell Cycle 2011;10:441-448.

45 Hou X, Tang Z, Liu H, Wang N, Ju H, Li K: Discovery of MicroRNAs associated with myogenesis by deep sequencing of serial developmental skeletal muscles in pigs. PLoS One 2012;7:e52123.

-46 Wang J, Song Y, Zhang Y, Xiao H, Sun Q Hou N, Guo S, Wang Y, Fan K, Zhan D, Zha L, Cao Y, Li Z, Cheng X, Zhang Y, Yang X: Cardiomyocyte overexpression of miR-27b induces cardiac hypertrophy and dysfunction in mice. Cell Res 2012;22:516-527. 